\title{
Les engagements anthropologiques vis-à-vis du développement
}

\section{Tania Murray Li}

Traducteur : Clara Maizi

\section{CpenEdition}

\section{Journals}

Édition électronique

URL : http://journals.openedition.org/anthropodev/497

DOI : 10.4000/anthropodev.497

ISSN : 2553-1719

Éditeur

APAD - Association pour l'anthropologie du changement social et du développement

Édition imprimée

Date de publication : 1 avril 2014

Pagination : 241-256

ISBN : 979-10-93476-00-1

ISSN : 2276-2019

Référence électronique

Tania Murray Li, « Les engagements anthropologiques vis-à-vis du développement », Anthropologie \& développement [En ligne], 37-38-39 | 2014, mis en ligne le 01 décembre 2016, consulté le 15 septembre 2020. URL : http://journals.openedition.org/anthropodev/497

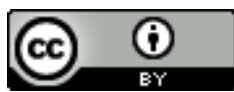

La revue Anthropologie \& développement est mise à disposition selon les termes de la Licence Creative Commons Attribution 4.0 International. 


\title{
Les engagements anthropologiques vis-à-vis du développement ${ }^{ \pm}$
}

\author{
Tania M. Li
}

Je propose de distinguer 3 types d'engagements anthropologiques face au développement, chacun d'entre eux étant accompagné d'un ensemble particulier de relations sur le terrain et de tensions caractéristiques. Pour moi, ces trois types d'engagements ne sont pas compatibles - ils ne sont pas connectés séquentiellement et ne sont habituellement pas conduits simultanément. D'où l'importance de nous situer, nous et nos pratiques, par rapport à eux.

Ces trois types sont :

- $\quad$ 1. L'anthropologie au service du développement programmé, de la planification, (que j'appellerai « programmation ») ou du Développement avec un « D » majuscule (Hart, 2009).

- 2. L'anthropologie comme engagement critique par rapport à la programmation.

- $\quad$ 3. L'anthropologie comme étude de conjonctures historiques spécifiques, leurs tendances et leurs frictions, et les luttes politiques émergeant dans ce contexte ; ou le développement avec un « $d$ » minuscule.

Je considère que le premier type - l'anthropologie au service du développement programmé - relève de façon générale d'une catégorie équivalente à celle de "gouvernementalité " élaborée par Foucault, ou de ce que j'appelle dans mon livre "la volonté d'améliorer " (Li, 2007). Autrement dit, c'est la tentative d'intervenir dans des processus sociaux, économiques, écologiques et autres, en vue de les ajuster ou de les rediriger dans une direction " améliorante » (Foucault, 1991a).

\footnotetext{
${ }^{1}$ Ce texte est la traduction de la Conférence donnée lors du Colloque de l'APAD de juin 2013 "Les relations d'enquête en contexte de développement ou d'urgence ". Nous remercions Clara Maizi et Philippe Lavigne Delville pour la traduction.
} 
J'appellerais cette pratique " la programmation " et tiens à souligner le type de connaissances particulières que cela requiert. Les programmeurs sont à la fois les personnes qui travaillent pour des agences transnationales, des ministères et des ONGs, et à la fois les spécialistes et les consultants (dont les anthropologues) qui les soutiennent.

Le deuxième type d'engagement anthropologique - critique - est intrinsèquement lié au premier. II consiste en un questionnement constant et vigilant de la pratique de gouverner. Qu'essayons-nous de gouverner ? Gouvernons-nous le bon objet, avons-nous la technique de gouvernement la plus efficace ? Gouvernons-nous trop ou pas assez ? Comment nos efforts pour gouverner fonctionnent-ils en pratique? La critique demande elle aussi des connaissances particulières.

Le troisième type d'engagement, que nous pourrions appeler l'ethnographie en tant que telle, n'est pas connecté à la programmation. C'est la tentative de comprendre le monde tel qu'il est, dans toute sa diversité, sa complexité et ses flux.

Je vais maintenant discuter de chacune de ces formes d'engagement plus en détail.

\section{Le travail de terrain au service de la programmation}

Le thème de cette conférence est " les relations d'enquête dans le contexte de développement ou d'urgence ". Ce sont deux contextes définis par un besoin urgent. Quelqu'un doit faire quelque chose : intervenir, résoudre un problème, apporter une amélioration. L'intervention exige sa propre forme de connaissance, qui est produite par deux pratiques. La première est la problématisation, c'est à dire l'identification des lacunes qui doivent être corrigées. La seconde est ce que j'appelle « rendering technical ", c'est-à-dire " transformer en objet susceptible d'une intervention technique ", techniciser au sens fort du terme. "Techniciser " est ainsi un raccourci pour l'ensemble des pratiques censées représenter "le domaine à gouverner comme un terrain intelligible avec des limites définissables et des caractéristiques particulières... définissant des frontières, rendant visible ce qu'il y a en leur sein, réunissant des informations sur ce 
qui est inclus et élaborant des techniques pour mobiliser les forces et les entités ainsi révélées »(Rose, 1999 : 33)².

Identifier le problème à résoudre et l'interpréter dans des termes techniques est souvent assez simple dans le contexte d'urgence, où les besoins immédiats viennent au premier plan. En ce qui concerne le développement, différents manques et lacunes ont pu être identifiés, notamment la pauvreté, les infrastructures précaires, la faible productivité, les institutions corrompues, le manque de voix et de choix, une mauvaise hygiène, une mauvaise nutrition, ou une faible sensibilisation à l'environnement. Par conséquent, avec le développement, il y a toujours place au débat pour savoir quels sont les problèmes les plus urgents et ceux qui le sont moins, quelles sont leurs causes et quelles sont leurs conséquences, et quelle est la meilleure façon d'y remédier.

Souvent, problème et solution voyagent ensemble. Un diagnostic de problème anticipe déjà la prescription-solution que le programmeur a en tête, ou qu'il peut de façon réaliste proposer à un organisme de financement. Par exemple, si le problème identifié est "la pauvreté », un programme de développement agricole pourrait spécifier « la faible productivité " comme cause de cette pauvreté, et proposer d'introduire de nouvelles semences et de nouvelles techniques d'irrigation. La pauvreté est " transformée en objet susceptible d'une intervention technique » dans les termes de ce que l'intervention anticipée peut effectivement proposer. Le manque de terres, pourtant fréquemment reconnu comme source de pauvreté rurale, ne peut être résolue par des moyens tels que de meilleures semences ou de meilleures techniques d'irrigation. Cela ne peut donc pas figurer dans cette chaîne de diagnostic/prescription.

Les anthropologues responsables de la programmation, qu'ils soient responsables de projets ou consultant, doivent s'engager dans des pratiques de problématisation et de technicisation. Fournir les types de connaissances pertinentes pour cela est une exigence professionnelle, une compétence nécessaire pour faire ce métier. La tension caractéristique pour l'anthropologue-comme-programmeur est principalement interne à

2 J'adapte le terme de "rendre susceptible de technique" à partir de Rose (p 79). Voir aussi Ferguson J., 1990. 
I'anthropologue. II nous faut en effet traduire notre connaissance dense et contextualisée des personnes, des lieux et des processus dans une matrice technique à la fois gérable et finançable. Cela peut faire violence aux relations que nous avons construites à travers notre travail sur le terrain, mais surtout nous trahissons notre propre connaissance complexe - sans doute pour une bonne cause.

J'ai pris conscience de ce genre de trahison quand j'ai entrepris une consultation en matière de droits fonciers en Indonésie pour l'Agence canadienne de développement lorsque cette dernière envisageait une intervention en la matière. Les exigences de programmation ont eu un effet viscéral sur moi. Je me suis rendue compte que j'allais devoir travailler à l'envers : je devais d'abord identifier une intervention plausible dans le cadre de l'expertise de l'agence, et seulement ensuite sélectionner et compiler les données et les analyses qui la soutiendraient. J'ai fait cela en dialogue avec des alliés dans le mouvement des droits fonciers qui ont beaucoup d'expérience en la matière. Ils savent comment repousser les limites, ouvrant le terrain à des politiques progressives (la création d'un chemin de fourmi, comme ils disent) tout en opérant dans les lignes de l'intelligibilité des bailleurs de fonds transnationaux ou des services de l'Etat. Néanmoins, les contraintes sont sévères. Si l'on admet trop de détails, ou si l'on met en évidence le rôle de certaines forces qu'une agence de développement ne peut pas contrôler, nos rapports seront mis à l'écart. C'est donc tout un art, et cela nécessite une certaine complicité : il faut un type particulier de relations d'enquête et de rapport au terrain pour construire une matrice technique avec une chaîne problème/solution plausible et finançable, matrice qu'il s'agit de défendre à l'aide de données pertinentes. Le livre de David Mosse, Cultivating Development (2005), décrit en détail les types de relations, compromis, et complicités que cela implique.

\section{L'anthropologie comme critique}

Lorsque nous analysons le travail de programmation, comme je l'ai fait dans The Will to Improve, nous rendons visibles les routines de problématisation et de technicisation, et nous les exposons à un examen critique. La critique est intrinsèque au fait de gouverner, comme Foucault l'a expliqué : il y a un va-et-vient constant entre le travail de conception des interven- 
tions gouvernementales, et le travail de questionnement de leurs diagnostics et leurs prescriptions. Néanmoins, le travail de terrain au service de la critique suscite souvent des relations tendues, car la critique peut être interprétée comme de l'hostilité ou comme une tentative de démolition. Elle peut également être interprétée comme une remise en question des compétences, comme lorsque la critique prétend comprendre des processus que les programmeurs et les acteurs du projet ne peuvent pas voir. Plutôt qu'une bataille de compétences, je vois cela comme une question de positionnement. Le travail de l'anthropologue en tant que programmeur demande des simplifications ainsi qu'une technicisation. C'est probablement aussi une exigence personnelle - une façon pour les programmeurs de développement de réussir à dormir la nuit. Face à l'impérieuse nécessité, l'urgence et la complexité, les programmeurs se fixent sur un type d'intervention qui est à leur portée. "Au moins nous faisons quelque chose d'utile - ou au moins nous essayons " (Mosse, 2011). La critique produit de l'inconfort et des réactions de défense : Êtes-vous indifférent à la souffrance? Si non, cessez de monter sur vos grands chevaux, et venez nous aider. Ou bien, vous ne devriez pas critiquer si vous n'avez pas de solution à proposer.

Les agences de développement sont habituées à la critique selon laquelle sous couvert de faire le bien, leurs interventions sont en fait une couverture pour l'exploitation et la domination. Je ne fais pas cette hypothèse. Je prends la volonté d'améliorer très au sérieux : je pense que beaucoup de programmeurs de développement veulent vraiment améliorer le monde. Le monde est plein d'interventions qui ne peuvent être comprises par le prisme de la domination ou du profit : les interventions pour bâtir des institutions communautaires, pour améliorer les moyens d'existence locaux, la santé et l'hygiène, et mettre en place une planification plus participative et démocratique en sont quelques exemples. Afin d'examiner ce type d'intervention de façon critique, il nous faut une approche différente, qui a des implications différentes sur les relations de terrain et sur les pratiques.

Je vais illustrer cela en résumant l'une des interventions que j'ai examinée dans mon livre: une intervention massive de plus d'un milliard de dollars conçue par des anthropologues faisant partie de l'équipe " développement social » au sein de la Banque Mondiale de Jakarta. Le pro- 
gramme avait un objectif particulièrement ambitieux : transformer la société indonésienne depuis le bas.

Cela commença par des études ethnographiques très détaillées de la vie au sein de villages indonésiens. C'était très loin de l'approche maladroite, " descendante " et insensible que l'on attribue habituellement à la Banque Mondiale ou aux autres agences bilatérales. Mais c'était des ethnographies d'un genre particulier. Pour s'intégrer de façon utile à la volonté d'améliorer, ces études devaient mettre au point une chaîne diagnostic/prescription dans laquelle la Banque pourrait de façon plausible jouer un rôle. Elles devaient identifier des problèmes techniques auxquels la Banque pourrait apporter des solutions techniques. Anticipant le fait que l'équipe de "développement social" trouverait sa mission technique dans l'amélioration de la "société», le problème identifié par les ethnographies financées par la Banque a été la mauvaise qualité de la planification du développement au niveau du village: corruption, manque de transparence, manque de participation. Ces déficiences aboutissaient à des décalages entre ce que les gens voulaient et ce que le gouvernement indonésien leur donnait. Pour combler cette lacune, la Banque entreprit de réformer la planification au niveau du village. L'idée était de se baser sur le capital social naturel et les capacités coutumières que l'on trouve dans les villages indonésiens, les restaurer, les optimiser, et les orienter vers d'autres finalités.

Malgré l'accent mis sur le capital social local, en pratique l'équipe du développement social de la Banque mis au point un épais manuel prévoyant en détail les procédures à suivre. Les règles furent soigneusement conçues, les experts du développement social ayant prévu de transformer les vieilles pratiques déficientes, et de les remplacer par de nouvelles, améliorées.

Chaque élément de ce manuel de règles avait un but éducatif. L'idée était que les gens apprendraient de nouvelles pratiques, réaliseraient à quel point elles fonctionnaient et les adopteraient comme les leurs. L'incitation était l'accès aux fonds du projet.

Par exemple, les villageois devaient s'engager dans un processus concurrentiel, très fortement organisé, afin d'accéder à des fonds pour les infrastructures du village. L'idée était que la participation à un tel processus 
allait diffuser de nouvelles compétences de planification. Plus précisément, puisque la concurrence favorisait les propositions les plus "pro-pauvres», les villageois apprendraient à penser de manière créative sur les types d'infrastructures réellement utiles aux pauvres.

Une autre exigence du programme était d'afficher le budget du projet sur le mur de la salle de réunion du village pour que chacun puisse voir combien d'argent avait été alloué et vérifier l'utilisation qui en est faite. Par cette pratique, les villageois apprendraient à penser aux fonds attribués par le projet comme à un droit qui leur était accordé de façon collective et qui devait être utilisé pour le bien commun, et non pas comme un cadeau ou une faveur qui pourrait disparaitre subrepticement comme cela arrivait souvent. Les experts de la Banque ont également effectué des études sur la culture de la corruption, et mis au point des interventions pour changer les points de vue des villageois, sur ce qui était acceptable et ce qui ne l'était pas. Ils ont complété leur approche culturelle par une tactique tirée de l'économie institutionnelle, en essayant de changer la structure d'incitation en augmentant les coûts et les risques, et en réduisant les récompenses. L'idée était de rééquilibrer l'équation de sorte que les comportements corruptifs ne seraient plus rationnels. A travers ces interventions et d'autres du même type, les programmeurs de la Banque exercèrent une volonté d'améliorer à un niveau extraordinairement détaillé. Ils fouillèrent dans les moindres détails de la vie du village pour éduquer les désirs, introduire de nouvelles pratiques, et transformer les conduites.

Sans surprise, leur programme ne fonctionna pas vraiment comme pré$\mathrm{vu}$. Les règles furent contournées. Mais il y eu tout de même quelques changements. Le plus bénéfique fut celui de la transparence sur le budget. Pour la première fois, en raison des règles de transparence de la Banque, les villageois savaient exactement combien d'argent avait été alloué à un projet tel que la construction de routes par exemple. Ainsi, si la route promise n'était pas achevée, ils savaient combien d'argent leur avait été volé. Stimulés par le sens de l'indignation que leurs nouvelles connaissances avaient activé, ils se mobilisèrent pour demander réparation. De victimes passives, ils sont devenus des citoyens actifs conscients de leurs droits, conscients du pouvoir qui leur permet de les exiger. Mais leur capacité à obtenir réparation a été limitée par les relations politiques et économiques 
obstinément inégales qui continuent encore à dominer la vie dans les villages indonésiens ${ }^{3}$.

Ma critique du programme porte sur le cœur de sa matrice technique, sa chaîne diagnostic - prescription. J'ai fait valoir que ce projet, financé par un prêt d'un milliard de dollars que les Indonésiens devraient à rembourser, a été mis en œuvre sous le label de la réduction de la pauvreté. Mais il ne s'affrontait pas aux processus en cours qui produisent la pauvreté en Indonésie, comme l'accaparement des terres coutumières par les élites indonésiennes, soutenues par l'armée et le capital transnational, pour l'extraction minière, l'exploitation du bois ou le développement des plantations. Même la pauvreté quotidienne n'a pas été affectée par ce programme. Une personne sans terre n'est pas aidée par un projet de construction d'une route d'accès aux fermes, car il n'a pas de ferme. Même les procédures impeccablement démocratiques pour la planification du développement au niveau du village ne sauraient aider cette personne, car la distribution des terres, ou la distribution de meilleurs salaires, ne sont pas au menu. L'ampleur et la répartition de la pauvreté, ainsi que les processus qui la produisent, restent donc inchangés.

Ma critique - qui souligne l'écart énorme entre ce que le programme a prétendu faire (réduire la pauvreté) et ce qu'il a effectivement fait (améliorer les procédures de planification villageoise) - a été troublante pour les programmeurs de la Banque parce qu'ils ne purent pas y répondre. Ils savaient trop bien qu'ils ne pouvaient pas aborder les questions politiques et économiques difficiles comme la distribution de terres ou la captation des ressources : la souveraineté nationale, l'étiquette diplomatique, ainsi que l'ensemble du langage du développement sur les "partenariats", avaient rendu cela impossible. La tension entre les anthropologuescomme-programmeurs de la Banque mondiale et une anthropologue basée à l'université en tant que critique, tous formés de manière similaire, produisit une impasse. Je pouvais respecter l'expertise professionnelle des anthropologues de la Banque et admirer leur courage et leur compétence, mais je ne pouvais pas accepter de les considérer comme des leaders en

\footnotetext{
${ }^{3}$ Voir par exemple la mobilisation sur une route que je décris dans Land's End: Capitalist Relations on an Indigenous Frontier, Duke University Press ( à paraître en août 2014).
} 
matière de réduction de la pauvreté parce que leur conception du programme, ainsi que la position structurelle de la Banque mondiale en Indonésie, rendaient cet objectif impossible.

Un membre de l'équipe du projet a suggéré que j'aurais dû faire plus de terrain. J'aurais dû passer du temps avec l'équipe de la Banque lors de leurs réunions et observer leurs débats et leurs négociations en coulisses. Je n'aurais alors pas fait autant attention aux documents du projet. Ne suis-je pas au courant que les documents ne sont que de simples mots, des artéfacts qui doivent être produits pour obtenir des fonds pour le projet ? Mais, répondis-je, sans ces documents, sans la matrice technique qui démontrait que le problème $A$ combiné à l'intervention $B$ conduirait à $C$, un résultat bénéfique, il n'y aurait pas eu un flux d'un milliard de dollars en prêt vers I'Indonésie. Que les objectifs aient été ou non atteints, la matrice en elle-même était déjà une intervention. Foucault l'admet volontiers, rien ne se passe jamais comme prévu dans les plans du programmeur. Pourtant, et il insiste là-dessus, "ce type de programmation n'était pas resté seulement une utopie dans la tête de quelques faiseurs de projet ". "Ces programmations de conduite, ces régimes de juridiction/véridiction ne sont pas des projets de réalité qui échouent. Ce sont des fragments de réalité ". "Ces programmations induisent toute une série d'effets dans le réel».». "Elles se cristallisent dans les institutions, elles informent les comportements des individus, elles servent de grilles pour la perception et l'appréciation des choses » (Perrot, $1980: 50)$. Ce ne sont, en bref, jamais de simples mots.

Pour approfondir le dialogue entre anthropologues situés différemment, j'aurais également pu souligner l'expérience de David Mosse, qui a écrit sur le travail en "arrière-boutique " des anthropologues-en-tantqu'experts du développement, un monde dont il a fait partie pendant plusieurs années. Il a décrit comment lui et ses collègues, dans une équipe de projet, ont produit des récits lisses et une cohérence politique à partir des réalités désordonnées auxquelles ils étaient confrontés. II souligne qu'ils ont fait cela non pas uniquement pour garder leurs emplois, mais aussi - et surtout - pour préserver les flux financiers qui leur permettaient de pouvoir continuer à produire des résultats bénéfiques. Mosse a écrit sur ce monde en tant qu'ancien membre de l'équipe du projet. II a reçu l'autorisation de ses anciens collègues pour changer de casquette et devenir cher- 
cheur, mais finalement ceux-ci furent furieux. II a écrit un brillant essai à ce sujet - au sujet de la tension entre les pratiques de terrain qui exigent des relations, une confiance, et la rupture nécessaire d'avec ces relations lorsque le chercheur lui-même s'éloigne, afin d'écrire sur ce qu'il a découvert (Mosse, 2006). Dans le but d'analyser le monde des praticiens, dont il avait fait partie, Mosse a dû le quitter. Ses anciens collègues, pour leur part, désiraient son retour dans le groupe, pour qu'ensemble ils puissent à nouveau concevoir un récit acceptable, tout comme ils l'avaient fait auparavant lors d'écritures de rapports pour les bailleurs de fonds de projet. Son refus leur a donné le sentiment d'être exposés et trahis. C'est donc une sorte d'incompatibilité : il est difficile et probablement impossible de faire partie d'une équipe de projet et d'écrire à ce sujet de manière ethnographique. Quels que soient les écrits de l'insider devenu outsider, ils seront probablement compris comme une divulgation inutile qui met en péril l'avenir du projet. Cela sera perçu comme une trahison, non seulement des relations collégiales, mais aussi de la volonté d'améliorer : un signe que l'insider devenu outsider ne se soucie pas du sort des pauvres, car il ne soutient pas les gens qui essaient de les aider.

Une autre incompatibilité, plus grave encore, concerne la relation entre la programmation et la critique. Les programmeurs tolèrent la critique tant que celle-ci peut être retraduite en programmation, ou utilisée pour «l'amélioration de l'amélioration », comme je l'ai appelée dans mon livre. La critique qui identifie les problèmes structurels auxquels les organismes de développement ne peuvent pas répondre est généralement considérée comme inutile, irréaliste, et donc sans valeur. Pourtant, je soutiens que la critique est une valeur en soi, et un rôle distinct des anthropologues en tant que critiques doit être défendu. "La critique, affirme Foucault (1991b : 84), ne doit pas avoir à faire la loi pour la loi. Il ne s'agit pas d'une étape dans une programmation. II s'agit d'un défi lancé à ce qui est " (Perrot, 1980 :53-54). Zygmunt Bauman (2007) défend ce point passionnément : à moins d'être satisfaits des choses comme elles sont, dit-il, nous devons être critiques. La critique, de son point de vue, est une position politique largement diffusée, elle n'est pas limitée aux savants ou aux experts ayant de nouveaux programmes à proposer. Ainsi toutes les critiques ne peuvent pas être intégrées à un nouveau programme, et d'ailleurs, elles n'ont pas à l'être. Si c'était le cas, nous devrions tirer la sonnette d'alarme : cela 
signifierait que l'anthropologue comme critique ne fait pas un travail suffisamment rigoureux et sérieux.

Que pouvons-nous faire alors de l'incompatibilité entre la programmation et la critique ? La réponse ne peut pas être d'abandonner l'une ou l'autre : les programmeurs et les critiques produisent différentes formes de connaissances, qui ont chacune de la valeur. Comme je l'ai expliqué précédemment, un élément central de la programmation est l'exigence selon laquelle les problèmes doivent être rédigés dans des termes qui se prêtent à des solutions techniques. Ce n'est pas un défaut. II doit en être ainsi. La programmation a besoin de fermeture : une décision de creuser ici pour s'attaquer à un problème et laisser un autre de côté. Mais l'ouverture intrinsèque à la critique ne peut être accomplie que lorsque les exigences de programmation sont suspendues, au moins pendant un certain temps. II est possible de se mettre d'accord sur une division respectueuse du travail. Ou bien, le même anthropologue peut être à la fois un programmeur et un critique, tant qu'elle / il garde les rôles distincts, et n'essaye pas de les occuper en même temps. Beaucoup d'entre nous ont deux casquettes, que nous portons selon les jours de la semaine, ou à différentes périodes de notre carrière. Des indications précieuses peuvent être tirées du franchissement de la frontière qui sépare la critique de la programmation, comme celui fait par David Mosse. En effet, l'un des apports les plus pénétrants de son étude a été d'exposer la frontière qui sépare ces deux mondes distincts, une limite assez importante pour être vigoureusement défendue par les anthropologues des deux côtés de la fracture.

Comment alors l'anthropologue-comme-critique peut-il répondre à l'affirmation selon laquelle il est froid, cynique, manque de compassion pour les gens qui souffrent et est peu disposé à aider à concevoir des programmes pour améliorer leur vie ? Ma réponse à cela est que la programmation est importante, mais qu'elle n'est pas la seule source de changement social. Mais alors qu'y a-t-il d'autre ? Cette question m'amène au troisième type d'engagement anthropologique pour le développement. 


\section{L'anthropologie comme étude de conjonctures historiques spécifiques, et des enjeux politiques qui s'en dégagent, à savoir le développement avec un petit « $d$ »}

Notre formation d'anthropologues nous donne les compétences nécessaires pour examiner les processus qui produisent la pauvreté, la violence, ou d'autres situations d'urgence, dans toute leur complexité et leur contexte spécifique. C'est un travail que nous devons maintenir. La pauvreté n'est pas une situation statique - elle est produite en continu, et parfois réduite grâce à des actions variées et dynamiques, dont beaucoup sont en dehors du champ d'application des programmes de développement. Par exemple, un changement dans les prix mondiaux des cultures particulières peut créer une nouvelle vague d'appauvrissement, ou au contraire apporter une certaine prospérité, selon ce que les agriculteurs cultivent, et les types de relations productives et extractives dans lequel ils sont impliqués. Sans une compréhension des processus qui produisent la pauvreté en un temps et un lieu particulier, il n'est pas possible de concevoir des interventions sur ce qui pourrait effectivement la réduire. Pourtant, les programmeurs de développement, contraints comme ils le sont de trouver des points techniques d'entrée pour leurs programmes, font rarement ce genre d'analyse.

Notre formation d'anthropologues nous permet aussi d'explorer le terrain de la politique et des enjeux de pouvoir au sens large. Il s'agit d'examiner les forces sociales qui reproduisent les inégalités, et les forces sociales susceptibles les perturber. Quels sont les groupes sociaux pertinents? Quels sont leurs intérêts, leurs alliances et leurs fractures ? Quels groupes se mobiliseraient pour changer le statu quo, quels autres se mobiliseraient pour le défendre ? Chaque conjoncture présente des ouvertures potentielles, des champs du possible qui peuvent être identifiés et élargis. Pourtant, je vois rarement ce type d'analyse dans les documents de politique de développement. Ceux-ci proposent aux gouvernements de faire ceci, aux communautés de faire cela, mais toujours avec très peu d'analyse des forces sociales pourtant nécessaires pour rendre possibles de tels changements. Le vide de données et d'analyses sur l'histoire, les rapports de force et les processus sociaux peut conduire à des attentes irréalistes, ou même au cynisme, à l'idée que rien ne changera jamais. 
Les militants ont tendance à être plus habiles que les chercheurs pour mener une analyse politique des forces sociales, car c'est une exigence centrale de leur métier. Certains activistes/chercheurs remarquables avec qui j'ai travaillé en Indonésie au fil des ans sont engagés politiquement à plusieurs niveaux : soutenir des villageois dans des luttes concrètes contre la saisie de terres ou leur éviction, rassembler différents groupes sociaux et plates-formes dans un front uni pour faire pression sur le gouvernement pour qu'il réponde aux revendications, tenter convaincre les politiciens et les fonctionnaires qu'eux-aussi seraient plus en sécurité et plus prospères si les injustices les plus flagrantes étaient traitées. Ils embrassent le rôle éducatif de l'intellectuel. Leurs écrits dans les médias sont dirigés principalement vers leur propre classe, car ils cherchent à repousser les limites de ce que les lecteurs de journaux considèrent comme normal, et les poussent à avoir honte de leur propre indifférence. Ils préparent également des critiques détaillées de lois, de politiques et de programmes d'intervention injustes, contestant le monopole des programmeurs sur la production de connaissances autorisées. Les anthropologues-comme-critiques peuvent être en mesure de contribuer aux efforts de ces savants-militants. Quelle que soit sa source, Foucault suggère que la critique est productive lorsqu'elle fonctionne comme " un instrument pour ceux qui luttent, résistent et ne veulent plus de ce qui est » (Perrot, $1980: 53$ ).

Des changements se produisent. La domestication du capitalisme sous la forme de l'État-providence dans l'Europe d'après-guerre est un exemple. Ce n'était pas le résultat de la seule planification, bien que les planificateurs aient joué un rôle. Cela prit des formes différentes selon les espaces nationaux, chacun avec sa propre constellation de forces sociales. C'était un compromis politique qui a duré trente ans, à peu près de 1948 à 1978, trente années au cours desquelles les conditions de vie du tiers le plus pauvre de la population se sont nettement améliorées, et le fossé entre riches et pauvres a diminué. À l'heure actuelle, nous nous dirigeons dans la direction opposée, avec des niveaux impressionnants d'inégalité à l'intérieur et entre les nations, ainsi qu'une nouvelle guerre contre les pauvres. Pourtant, il existe des contre-tendances. La recherche anthropologique est une fois encore pertinente. Nous n'avons pas besoin de généraliser ou d'être grandiose, mais plutôt de nous demander - de manière précise et spécifique - si quelque chose de progressiste se passe, pourquoi est-ce que 
cela se passe ici ? Pourquoi maintenant ? Pourquoi sous cette forme et pas sous une autre? (Li, 2009; Ferguson, 2009). Inversement, si ce qui émerge est l'appauvrissement et l'abandon, ou la violence et les attaques xénophobes, les anthropologues peuvent chercher à expliquer ceci : quelles sont les configurations sociales et culturelles qui permettent l'existence de ces tendances, ou les rendent acceptable ? Quelles sont les configurations spatiales, les flux d'information, les histoires que les gens racontent, les représentations populaires qui séparent les méritants de ceux qui ne méritent rien, le citoyen de l'étranger, nous d'eux ? Ce type d'anthropologie se concentre sur ce qui émerge, et ce faisant, elle remet la programmation à sa place, en tant qu'une force sociale parmi d'autres qui font changer notre monde. Nous devrions prêter attention à la programmation, car elle produit des effets, mais nous ne devrions pas la placer au centre car ce n'est pas nécessairement l'élément le plus important de la constellation.

Quelles sont alors les tensions liées à ce type de recherches de terrain recherches que nous pourrions faire " dans un contexte de développement ou d'urgence ", mais qui ne soient pas centrées sur l'intervention ? La tension habituelle porte sur la pertinence. Les programmeurs et les critiques étroitement associés à la programmation ne voient pas ce que représente ce qu'ils pourraient appeler recherche "pure» ou "scientifique». Ils n'en voient pas l'intérêt. Si un rapport ne se finit pas par une conclusion qui dit : donc le gouvernement devrait faire $x$, ou la politique devrait être $y$ ou la réponse est $z$, ils pensent qu'il n'a pas de valeur. Par conséquent, il peut être difficile de trouver des financements pour ce type de recherche, en particulier dans des contextes où les agences de développement sont la principale ou la seule source de financement de la recherche. Les gouvernements - y compris le gouvernement du Canada - réduisent les fonds pour la recherche "pure» en faveur de la recherche qui peut être appliquée ou instrumentalisée. C'est une contrainte énorme. Cela peut de plus créer des tensions dans le travail de terrain. Les villageois peuvent se demander : si vous n'êtes pas là pour nous aider, ou nous apporter des projets, ou nous donner de l'argent ou des emplois, alors pourquoi êtes-vous ici ? Les parties du monde qui ont été saturées par les programmes de développement et par la recherche instrumentale sont devenues des territoires difficiles pour la recherche ethnographique qui cherche à explorer des modalités 
émergentes de politiques et des changements sociaux sans les rattacher à des prescriptions.

\section{Conclusion}

J'ai distingué trois types d'engagements anthropologiques par rapport au développement : l'anthropologie comme soutien à la programmation, l'anthropologie comme critique, et l'anthropologie comme étude des processus sociaux et de l'émergence. Chacun a des tensions intrinsèques qui lui sont propres, et il existe entre eux des gouffres qui ne peuvent pas ou ne doivent pas être franchis. Je ne dis pas qu'un type de pratique est supérieur aux deux autres, ou qu'il doit les remplacer. Je fais simplement valoir que la reconnaissance de leur caractère distinct nous aide à comprendre les types de connaissances, de relations de travail sur le terrain, et de tensions propres à chacun. Un programmeur ne peut pas être dans le même temps un critique, ni faire plein usage de sa connaissance des forces sociales qui façonnent le monde : il doit tracer une frontière et rendre les problèmes techniques afin de faire son travail. Un critique se consacre à exposer les lacunes des programmes, à perforer leurs frontières, contester les suffisances et les illusions, mais il y a une limite à cet exercice : à la fin de la journée, la programmation nécessite une fermeture et les apports d'un critique sera soit acceptée soit rejetée. Les études centrées sur l'émergence n'aboutissent pas à des programmes d'intervention parce qu'elles se centrent sur la spécificité de la conjoncture et les relations politiques, économiques et sociales qui la composent. Ces relations sont propres à un moment et un lieu, et ne peuvent être reproduites; il n'est pas facile ni de les exploiter ni de les contrôler. Pourtant, il nous faut les étudier, car ce sont les relations qui façonnent et changent notre monde, pour le meilleur et pour le pire.

\section{Bibliographie}

BAUMAN, Z., 2007, "Has the Future a Left?", Soundings 35. 
Ferguson, J., 1994, The Anti-Politics Machine: "Development," Depoliticization, and Bureaucratic Power in Lesotho, Minneapolis, University of Minnesota Press.

Ferguson, J., 2009, "The Uses of Neoliberalism", Antipode 41, n S1 : 166-84.

Foucault, M., 1980, in Perrot M. ed. L'impossible prison, recherches sur les systèmes pénitenciaires au XIX ${ }^{\circ}$ siècle, Paris, Seuil.

Hart, G., 2009, "D/Developments after the Meltdown.", Antipode 41, n S1 : 117-41.

Li, T.M., 2007, The Will to Improve: Governmentality, Development, and the Practice of Politics, Durham NC, Duke.

Li, T.M., 2009, "To Make Live or Let Die? Rural Dispossession and the Protection of Surplus Populations", Antipode 41, n S1 : 63-93.

Li, T.M., 2014, Land's End: Capitalist Relations on an Indigenous Frontier, Durham NC, Duke.

Mosse, D., 2005, Cultivating Development: An Ethnography of Aid Policy and Practice, London, Pluto Press.

Mosse, D., 2006, "Anti-Social Anthropology? Objectivity, Objection and the Ethnography of Public Policy and Professonal Communities", Journal of the Royal Anthropological Institute 12 (4) : 935-56.

Mosse, D. ed., 2011, Adventures in Aidland: The Anthropology of Professionals in International Development, New York, Berghahn

Perrot, M. (Ed.), 1980, L'impossible prison: recherches sur le système pénitentiaire au XIXe siècle, Paris, Editions du Seuil. .

Rose, N., 1999, Powers of Freedom: Reframing Political Thought, Cambridge, Cambridge University Press.

\section{Aviviviv}

Tania Murray Li is Professeur of Anthropology at the University of Toronto E-mail : tania.li@utoronto.ca 